\title{
sciendo
}

Int. J. of Applied Mechanics and Engineering, 2020, vol.25, No.3, pp.158-168

DOI: $10.2478 /$ ijame-2020-0040

\section{ANALYSIS OF TIME-FRACTIONAL HEAT TRANSFER AND ITS THERMAL DEFLECTION IN A CIRCULAR PLATE BY A MOVING HEAT SOURCE}

\author{
S. THAKARE* and M. WARBHE \\ Department of Mathematics, Sarvodaya Mahavidyalaya \\ Sindewahi, Chandrapur, INDIA \\ E-mail: shivthakre5@gmail.com
}

\begin{abstract}
Mathematical modeling of a thin circular plate has been made by considering a nonlocal Caputo type time fractional heat conduction equation of order $0<\alpha \leq 2$, by the action of a moving heat source. Physically convective heat exchange boundary conditions are applied at lower, upper and outer curved surface of the plate. Temperature distribution and thermal deflection has been investigated by a quasi-static approach in the context of fractional order heat conduction. The integral transformation technique is used to analyze the analytical solution to the problem. Numerical computation including the effect of the fractional order parameter has been done for temperature and deflection and illustrated graphically for an aluminum material.
\end{abstract}

Key words: fractional order, circular plate, integral transform, Mittag-Leffler function, thermal stresses.

\section{Introduction}

The classical Fourier law of heat conduction relates heat flow and the temperature change of a solid. It is observed that the above law is not so accurate for many physical situations and materials like amorphous, dielectrics, polymers, colloids, glassy, transient loading, etc. This leads to the generalization of thermoelasticity in which a fractional heat conduction model of non-integer order derivative is involved. This fractional heat conduction model is more useful in physical problems as compared to the classical approach. Povstenko [1] investigated a quasi-static uncoupled theory of thermoelasticity which is based on the timefractional heat conduction equation of order $\alpha$. Also, he derived the expression of thermal stresses for a onedimensional and two-dimensional Cauchy problem. Povstenko [2] generalized the Fourier law and studied the thermal stress behaviour based on the heat conduction equation involving time and space fractional order derivatives. Youssef [3] constructed a new model of thermoelastic theory in the context of fractional order heat conduction, as well as proved its uniqueness theorem. Further, he studied a one dimensional thermally shocked problem for a half-space made of an elastic material by using the Laplace transform and state-space techniques.

Povstenko [4] investigated thermal behaviour in an infinite body by using the concept of Caputo time-fractional order derivative. Jiang and $\mathrm{Xu}$ [5] used the theory of fractional calculus to obtain a time fractional Fourier law and built a time-fractional derivative heat conduction equation in the orthogonal curvilinear coordinate system. Ezzat [6] constructed a new model which is based on the theory of magnetothermoelasticity in the context of fractional order derivative. He also solved a one-dimensional thermoelectric elastic problem for half space by using state-space and the Laplace transform technique. Povstenko [7] formulated the theory of thermal stresses by following (Compte and Metzler) generalized Cattaneo-type equations with time-fractional derivatives. Ezzat [8] did mathematical modeling of the thermoelectric MHD theory in the context of fractional orders heat conduction and solved Stokes' first

\footnotetext{
* To whom correspondence should be addressed
} 
problem with heat source by the application of the Laplace transform and state space techniques. Ezzat and Ezzat [9] constructed a new mathematical model of poro-thermoelasticity in the context of fractional order heat conduction. The Laplace transform is applied to solve a one-dimensional problem of a poroelastic half space subjected to heating. Ezzat and El-Bary [10] developed a fractional derivative heat transfer mathematical model for generalized magneto-thermoelasticity and establish some coupled and generalized thermoelasticity theories. Xiong and Niu [11] established a generalized thermoelastic diffusion theory of fractional-order for an anisotropic and linearly thermoelastic diffusive media.

Recently, Kumar and Khobragade [12 and 13] found temperature and thermal stress for a circular disk due to action of partially distributed heat supply in the context of the time fractional order theory of thermoelasticity. Kumar and Khobragade [14 and 15] studied thermoelastic behavior for a solid circular cylinder by application of the time fractional order derivative theory. Some other related significant contributions to the thermoelastic theory has been found in [18- 31].

In this article, mathematical modeling of a thin circular plate subjected to the action of a moving heat source has been done by considering the nonlocal Caputo type time fractional heat conduction equation of order $0<\alpha \leq 2$. The expression of temperature and thermal deflection has been investigated by a quasi-static approach in the context of the fractional order heat conduction by integral transform technique. Numerical computation has been done for temperature and deflection and the effect of fractional order parameter was illustrated graphically for an aluminum material. To the best of the author's knowledge till date no one has conducted a study on thermal deflection using fractional heat conduction in a thin circular plate by the action of a moving heat source. This is a new contribution to the field.

\section{Problem formulation}

In this problem we consider a homogeneous isotropic thermoelastic thin circular plate subjected to the moving heat source of thickness $h$ and radius $b$. We shall assume the heat source moves on the plate surface along a circular trajectory at radius round the centre of the plate with constant angular velocity by following [16].

The expression for the Caputo type fractional derivative of function $f(t)$ is given as [24]

$$
\frac{d^{\alpha} f(t)}{d t^{\alpha}}=\frac{1}{\Gamma(n-\alpha)} \int_{0}^{t}(t-\tau)^{n-\alpha-1} \frac{d^{n} f(\tau)}{d \tau^{n}} d \tau, \quad t>0, \quad n-1<\alpha<n,
$$

with the following Laplace transform rule, where the Caputo derivative needs the initial values of the function $f(t)$ and its corresponding integral derivatives of order $k=1,2,3, \ldots, n-1$

$$
L\left\{\frac{d^{\alpha} f(t)}{d t^{\alpha}}\right\}=s^{\alpha} L\{\bar{f}(s)\}-\sum_{k=0}^{n-1} f^{(k)}\left(0^{+}\right) s^{\alpha-1-k}, \quad n-1<\alpha<n
$$

in which $s$ is the transform parameter and $n$ is a positive integer.

\subsection{Temperature distribution}

The temperature distribution of the circular plate is described by the differential equation of heat conduction (following [17] and [24]) in the context of the fractional-order theory subjected to a time dependent heat flux in a circular plate as

$$
\frac{\partial^{2} \theta}{\partial r^{2}}+\frac{1}{r} \frac{\partial \theta}{\partial r}+\frac{1}{r^{2}} \frac{\partial^{2} \theta}{\partial \varphi^{2}}+\frac{\partial^{2} \theta}{\partial z^{2}}+\frac{1}{k} g(r, \varphi, z, t)=\frac{1}{a} \frac{\partial^{\alpha} \theta}{\partial t^{\alpha}}
$$


where $\theta(r, \varphi, z, t)$ denote temperature, $k$ denotes thermal conductivity, $a$ denotes thermal diffusivity and $g(r, \varphi, z, t)$ denotes a volumetric energy generation.

Further, it is assumed that the moving heat source provides thermal energy. The function $g(r, \varphi, z, t)$, in Eq.(2.3) has the form

$$
g(r, \varphi, z, t)=T_{0} \delta\left(r-r_{0}\right) \delta(\varphi-\phi(t)) \delta\left(z-z_{0}\right)
$$

where $T_{0}$ represents the stream of the heat, $r_{0}$ refers to the radius of the circular trajectory along which the heat source move, $\delta()$ denotes the Dirac delta function and $\phi(t)$ denotes the function describing the movement of the heat source and is defined as

$$
\phi(t)=\omega t
$$

where $\omega$ denotes the angular velocity of the moving heat source

Using the physically convective heat exchange boundary conditions at the surface by following [24] we can write

$$
\begin{aligned}
& \left.K D_{R L}^{l-\alpha} \frac{\partial \theta}{\partial r}\right|_{r=b}=\alpha_{0}\left(\theta_{0}-\theta\right) ; t>0, \\
& \left.K D_{R L}^{I-\alpha} \frac{\partial \theta}{\partial z}\right|_{z=h}=\alpha_{0}\left(\theta_{0}-\theta\right) ; t>0, \\
& \left.K D_{R L}^{l-\alpha} \frac{\partial \theta}{\partial z}\right|_{z=0}=\alpha_{0}\left(\theta_{0}-\theta\right) ; t>0,
\end{aligned}
$$

and initial conditions,

$$
\begin{aligned}
& \theta=0, \quad \text { at } \quad t=0, \quad 0<\alpha \leq 2, \\
& \frac{\partial \theta}{\partial t}=0, \quad \text { at } \quad t=0, \quad 1<\alpha \leq 2
\end{aligned}
$$

where $\alpha_{0}$ denotes the convective heat transfer coefficient, $K$ is the heat transfer coefficient, $\theta_{0}$ is the known temperature of the surrounding medium, Also, $D_{R L}^{-\alpha} \theta(r, \varphi, z, t)$ for $\alpha>0$ is the Riemann-Liouville fractional integral $I^{\alpha} \theta(r, \varphi, z, t)$.

\subsection{Thermal defection}

The deflection function $w(r, \varphi, t)$, satisfies the following differential equation (cf. [22]) which is defined as

$$
\nabla^{4} \omega=-\frac{\nabla^{2} M_{\theta}}{D(1-v)}
$$


where $M_{\theta}$ denotes the thermal moment of the plate and is defined as

$$
M_{\theta}=a_{t} E \int_{0}^{h} \theta(r, \varphi, z, t) z d z
$$

Here $D$ represents the flexural rigidity of the thin circular plate denoted as

$$
D=\frac{E h^{3}}{12\left(1-v^{2}\right)} .
$$

In Eq.(2.12), $a_{t}$ denotes the coefficients of the linear thermal expansion, $E$ refers to the Young's modules and $v$ represents the Poisson's ratio of the plate material, respectively.

For out-of-plane deformation, the boundary conditions are given as

$$
\omega=0 \quad \text { at } \quad r=0 .
$$

\section{Solution of the problem}

\subsection{Determination of the transient heat conduction problem}

In order to find the expression for the temperature distribution function $\theta(r, \varphi, z, t)$ we first define the finite Fourier integral transform and its inverse transform over the variable $z$ as [23]

$$
\begin{aligned}
& \bar{\theta}\left(r, \varphi, \eta_{p}, t\right)=\int_{z^{\prime}=0}^{h} K\left(\eta_{p}, z^{\prime}\right) \theta\left(r, \varphi, z^{\prime}, t\right) d z^{\prime} \\
& \theta(r, \varphi, z, t)=\sum_{p=1}^{\infty} K\left(\eta_{p}, z\right) \bar{\theta}\left(r, \varphi, \eta_{p}, t\right) .
\end{aligned}
$$

With Kernel of the transformation

$$
K\left(\eta_{p}, z\right)=\frac{\sqrt{2}\left(\eta_{p} \cos \eta_{p} z+\frac{\alpha_{0}}{k} \sin \eta_{p} z\right)}{\sqrt{A B+\frac{\alpha_{0}}{k}}}
$$

where

$$
A=\eta_{p}{ }^{2}+\left(\frac{\alpha_{0}}{k}\right)^{2} \quad \text { and } \quad B=h+\alpha_{0}\left[\frac{k}{\eta_{p}{ }^{2} k^{2}-\alpha_{0}^{2}}\right]
$$

where $\eta_{p}{ }^{\prime} s$ are the roots of the transcendental equation

$$
\tan \eta_{p} h=\frac{2\left(\frac{\alpha_{0}}{k}\right) \eta_{p}}{\eta_{p}{ }^{2}-\left(\frac{\alpha_{0}}{k}\right)^{2}}
$$




$$
\begin{aligned}
& \overline{\bar{\theta}}\left(r, v^{\prime}, \eta_{p}, t\right)=\int_{\varphi^{\prime}=0}^{2 \pi} \cos v^{\prime}\left(\varphi-\varphi^{\prime}\right) \bar{\theta}\left(r, \varphi^{\prime}, \eta_{p}, t\right) d \varphi^{\prime}, \\
& \bar{\theta}\left(r, \varphi, \eta_{p}, t\right)=\frac{1}{\pi} \sum_{v^{\prime}=0}^{\infty} \overline{\bar{\theta}}\left(r, v^{\prime}, \eta_{p}, t\right) .
\end{aligned}
$$

Further, we define the finite Hankel integral transform and its inverse transform over the variable $r$ as [23]

$$
\begin{aligned}
& \overline{\bar{\theta}}\left(\beta_{m}, v^{\prime}, \eta_{p}, t\right)=\int_{r^{\prime}=0}^{b} K_{0}\left(\beta_{m}, r^{\prime}\right) \overline{\bar{\theta}}\left(r^{\prime}, v^{\prime}, \eta_{p}, t\right) d r^{\prime}, \\
& \overline{\bar{\theta}}\left(r, v^{\prime}, \eta_{p}, t\right)=\sum_{m=1}^{\infty} K_{0}\left(\beta_{m}, r\right) \overline{\bar{\theta}}\left(\beta_{m}, v^{\prime}, \eta_{p}, t\right) .
\end{aligned}
$$

With the Kernel of transform is

$$
K_{0}\left(\beta_{m}, r\right)=\frac{\sqrt{2}}{b} \frac{1}{\left[1+\left(\frac{\alpha_{0}}{k \beta_{m}}\right)^{2}\right]^{1 / 2}} \frac{J_{0}\left(\beta_{m} r\right)}{J_{0}^{\prime}\left(\beta_{m} b\right)},
$$

and $\beta_{1}, \beta_{2}, \beta_{3}$, are the positive roots of the transcendental equation

$$
J_{0}^{\prime}\left(\beta_{m} b\right)=\frac{\alpha_{0}}{k \beta_{m}} J_{0}\left(\beta_{m} b\right)
$$

Now, applying the finite Fourier, finite Hankel and Laplace transform and their inversions to Eq.(2.3) and using the transformed initial and boundary conditions defined in Eqs (2.6)-(2.10), we obtain the expression of the temperature distribution function as

$$
\begin{aligned}
& \theta(r, \varphi, z, t)=\frac{a}{k \pi} \sum_{m=1}^{\infty} \sum_{p=1}^{\infty} \sum_{v^{\prime}=1}^{\infty} K_{0}\left(\beta_{m}, r\right) K\left(\eta_{p}, z\right) \times \\
& \times\left\{\left[T_{0} r_{0} K_{0}\left(\beta_{m}, r_{0}\right) \cos v^{\prime}(\phi-\varphi(t)) K\left(\eta_{p}, z_{0}\right)\right]+\right. \\
& +\alpha_{0} \theta_{0}\left[\frac{-b k d K_{0}\left(\beta_{m}, b\right)}{d r} \int_{z^{\prime}=0}^{h} \int_{\phi^{\prime}=0}^{2 \pi} K\left(\eta_{p}, z^{\prime}\right) \cos v^{\prime}\left(\phi-\phi^{\prime}\right) d z^{\prime} d \phi^{\prime} \cdot\right. \\
& \left..\left(\left\{K\left(\eta_{p}, 0\right)+K\left(\eta_{p}, h\right)\right\} \int_{r^{\prime}=0}^{b} \int_{\phi^{\prime}=0}^{2 \pi} r^{\prime} K_{0}\left(\beta_{m}, r^{\prime}\right) \cos v^{\prime}\left(\phi-\phi^{\prime}\right) d r^{\prime} d \phi^{\prime}\right)\right]\left[E_{\alpha}\left(-a\left(\beta_{m}^{2}+\eta_{p}^{2}\right) t^{\alpha}\right]\right\}
\end{aligned}
$$

where $L^{-1}\left[\frac{1}{s^{\alpha}+a\left(\beta_{m}^{2}+\eta_{p}^{2}\right)}\right]=E_{\alpha}\left(-a\left(\beta_{m}^{2}+\eta_{p}^{2}\right) t^{\alpha}\right.$.

Here $E_{\alpha}($.$) represents the Mittag-Leffler function.$ 


\subsection{Determination of thermal deflection $w(r, \varphi, t)$}

Using Eq.(3.9) into Eq.(2.12), one obtains

$$
\begin{aligned}
& M_{\theta}=-\sqrt{2 k} a_{t} E \frac{a}{k \pi} \sum_{m=1}^{\infty} \sum_{n=1}^{\infty} \sum_{p=1}^{\infty} K_{0}\left(\beta_{m}, r\right) \frac{\left(\eta_{p}^{2} \sin \eta_{p} z-\frac{\alpha_{0}}{k} \eta_{p} \cos \eta_{p} z\right)}{\sqrt{k A B+\alpha_{0}}} \times \\
& \times\left\{\left[T_{0} r K_{0}\left(\beta_{m}, r_{0}\right) \cos v^{\prime}(\phi-\varphi(t)) K\left(\eta_{p}, z_{0}\right)\right]+\right. \\
& +\alpha_{0} \theta_{0}\left[\frac{-b k d K_{0}\left(\beta_{m}, b\right)}{d r} \int_{z^{\prime}=0}^{h} \int_{\phi^{\prime}=0}^{2 \pi} K\left(\eta_{p}, z^{\prime}\right) \cos v^{\prime}\left(\phi-\phi^{\prime}\right) d z^{\prime} d \phi^{\prime}\right] . \\
& .\left(\left\{K\left(\eta_{p}, 0\right)+K\left(\eta_{p}, h\right)\right\} \int_{r^{\prime}=0}^{b} \int_{\phi^{\prime}=0}^{2 \pi} r^{\prime} K_{0}\left(\beta_{m}, r^{\prime}\right) \cos v^{\prime}\left(\phi-\phi^{\prime}\right) d r^{\prime} d \phi^{\prime}\right)\left[E_{\alpha}\left(-a\left(\beta_{m}^{2}+\eta_{p}^{2}\right) t^{\alpha}\right] .\right.
\end{aligned}
$$

Assuming the solution of Eq.(2.11), satisfying condition (2.14) as

$$
\omega=\sum_{m=1}^{\infty} C_{m} \frac{J_{0}\left(\beta_{m} r\right)}{J_{0}^{\prime}\left(\beta_{m} b\right)}
$$

where $\beta_{m}^{\prime}$ are the positive roots of the transcendental equation

$$
J_{0}^{\prime}\left(\beta_{m} b\right)=\frac{\alpha_{0}}{k \beta_{m}} J_{0}\left(\beta_{m} b\right) .
$$

Hence solution (3.11) satisfies condition (2.14).

Now, we obtain

$$
\nabla^{4} \omega=\sum_{m=1}^{\infty} C_{m} \beta_{m}^{4} \frac{J_{0}\left(\beta_{m} r\right)}{J_{0}^{\prime}\left(\beta_{m} b\right)} .
$$

Also,

$$
\begin{aligned}
& \nabla^{2} M_{\theta}=\sqrt{2 k} a_{t} E \frac{a}{k \pi} \sum_{m=1}^{\infty} \sum_{n=1}^{\infty} \sum_{p=1}^{\infty} \beta_{m}^{2} K_{0}\left(\beta_{m}, r\right) \frac{\left(\eta_{p}^{2} \sin \eta_{p} z-\frac{\alpha_{0}}{k} \eta_{p} \cos \eta_{p} z\right)}{\sqrt{k A B+\alpha_{0}}} \times \\
& \times\left\{\left[T_{0} r K_{0}\left(\beta_{m}, r_{0}\right) \cos v^{\prime}(\phi-\varphi(t)) K\left(\eta_{p}, z_{0}\right)\right]+\right. \\
& +\alpha_{0} \theta_{0}\left[\frac{-b k d K_{0}\left(\beta_{m}, b\right)}{d r} \int_{z^{\prime}=0}^{h} \int_{\phi^{\prime}=0}^{2 \pi} K\left(\eta_{p}, z^{\prime}\right) \cos v^{\prime}\left(\phi-\phi^{\prime}\right) d z^{\prime} d \phi^{\prime}\right] . \\
& .\left(\left\{K\left(\eta_{p}, 0\right)+K\left(\eta_{p}, h\right)\right\} \int_{r^{\prime}=0}^{b} \int_{\phi^{\prime}=0}^{2 \pi} r^{\prime} K_{0}\left(\beta_{m}, r^{\prime}\right) \cos v^{\prime}\left(\phi-\phi^{\prime}\right) d r^{\prime} d \phi^{\prime}\right)\left[E_{\alpha}\left(-a\left(\beta_{m}^{2}+\eta_{p}^{2}\right) t^{\alpha}\right] .\right.
\end{aligned}
$$

Substituting Eqs (3.11) and (3.13) into Eq.(2.11) and on simplifying, one obtains 


$$
\begin{aligned}
& C_{m}=-\sqrt{2 k} \frac{a_{t} E}{D(1-v)} \frac{a}{k \pi} \sum_{m=1}^{\infty} \sum_{n=1}^{\infty} \sum_{p=1}^{\infty} \frac{1}{\beta_{m}^{2}} \frac{\sqrt{2}}{b} \frac{1}{\left[1+\left(\frac{\alpha_{0}}{k \beta_{m}}\right)^{2}\right]^{1 / 2}} \frac{\left(\eta_{p}^{2} \sin \eta_{p} z-\frac{\alpha_{0}}{k} \eta_{p} \cos \eta_{p} z\right)}{\sqrt{k A B+\alpha_{0}}} \times \\
& \times\left\{\left[T_{0} r K_{0}\left(\beta_{m}, r_{0}\right) \cos v^{\prime}(\phi-\varphi(t)) K\left(\eta_{p}, z_{0}\right)\right]+\right. \\
& +\alpha_{0} \theta_{0}\left[\frac{-b k d K_{0}\left(\beta_{m}, b\right)}{d r} \int_{z^{\prime}=0}^{h} \int_{\phi^{\prime}=0}^{2 \pi} K\left(\eta_{p}, z^{\prime}\right) \cos v^{\prime}\left(\phi-\phi^{\prime}\right) d z^{\prime} d \phi^{\prime}\right] . \\
& \cdot\left(\left\{K\left(\eta_{p}, 0\right)+K\left(\eta_{p}, h\right)\right\} \int_{r^{\prime}=0}^{b} \int_{\phi^{\prime}=0}^{2 \pi} r^{\prime} K_{0}\left(\beta_{m}, r^{\prime}\right) \cos v^{\prime}\left(\phi-\phi^{\prime}\right) d r^{\prime} d \phi^{\prime}\right)\left[E_{\alpha}\left(-a\left(\beta_{m}^{2}+\eta_{p}^{2}\right) t^{\alpha}\right] .\right.
\end{aligned}
$$

Substituting Eq.(3.14) into Eq.(3.11), one obtains

$$
\begin{aligned}
& \omega=-2 \sqrt{k} \frac{a_{t} E}{D(1-v)} \frac{a}{k \pi} \sum_{m=1}^{\infty} \sum_{n=1}^{\infty} \sum_{p=1}^{\infty} \frac{1}{\beta_{m}^{2}} \frac{1}{b} \frac{1}{\left[1+\left(\frac{\alpha_{0}}{k \beta_{m}}\right)^{2}\right]^{1 / 2}} \frac{J_{0}\left(\beta_{m} r\right)}{J_{0}^{\prime}\left(\beta_{m} b\right)} \frac{\left(\eta_{p}^{2} \sin \eta_{p} z-\frac{\alpha_{0}}{k} \eta_{p} \cos \eta_{p} z\right)}{\sqrt{k A B+\alpha_{0}}} \times \\
& \times\left\{\left[T_{0} r K_{0}\left(\beta_{m}, r_{0}\right) \cos v^{\prime}(\phi-\varphi(t)) K\left(\eta_{p}, z_{0}\right)\right]+\right. \\
& +\alpha_{0} \theta_{0}\left[\frac{-b k d K_{0}\left(\beta_{m}, b\right)}{d r} \int_{z^{\prime}=0}^{h} \int_{\phi^{\prime}=0}^{2 \pi} K\left(\eta_{p}, z^{\prime}\right) \cos v^{\prime}\left(\phi-\phi^{\prime}\right) d z^{\prime} d \phi^{\prime}\right] . \\
& \cdot\left(\left\{K\left(\eta_{p}, 0\right)+K\left(\eta_{p}, h\right)\right\} \int_{r^{\prime}=0}^{b} \int_{\phi^{\prime}=0}^{2 \pi} r^{\prime} K_{0}\left(\beta_{m}, r^{\prime}\right) \cos v^{\prime}\left(\phi-\phi^{\prime}\right) d r^{\prime} d \phi^{\prime}\right)\left[E_{\alpha}\left(-a\left(\beta_{m}^{2}+\eta_{p}^{2}\right) t^{\alpha}\right] .\right.
\end{aligned}
$$

\section{Numerical results and discussions}

An aluminum plate has been chosen for numerical computations.

The radius of the circular plate is $b=1 \mathrm{~m}$, thickness of the circular plate $h=0.2 \mathrm{~m}$, angular velocity $\omega=2 \pi, t=5 \mathrm{sec}$, heat transfer coefficient $\alpha_{o}=10$, temperature of the surrounding medium $\theta_{0}=20^{\circ} \mathrm{C}$, $r_{0}=0.5, z_{0}=0.1$, thermal conductivity $k=0.86\left(\mathrm{~m}^{2} \mathrm{~s}^{-1}\right)$. Thermal diffusivity $a=84.18 \times 10^{-6}\left(\mathrm{~m}^{2} \mathrm{~s}^{-1}\right)$, density $\rho=2707 \mathrm{~kg} / \mathrm{m}^{3}$, specific heat $c_{p}=896 \mathrm{~J} / \mathrm{kg} \mathrm{K}$, Poisson ratio $v=0.35$, coefficient of linear thermal expansion $\alpha_{t}=22.10 \times 10^{-6} \frac{1}{K}$, Lamé constant $\mu=26.67$.

The positive roots of the transcendental Eq.(3.8) are $\beta_{1}=3.005, \beta_{2}=6.772, \beta_{3}=10.06$, $\beta_{4}=13.258, \beta_{5}=16.428$ and the positive roots of the transcendental Eq.(3.4) are $\eta_{1}=20.805$, $\eta_{2}=34.654, \eta_{3}=49.435, \eta_{4}=64.613, \eta_{5}=79.985$.

MATEMATICA software is used for numerical computations of the thermoelastic problem under consideration. All the obtained solutions of temperature distribution and thermal deflection are plotted along 
the radial direction by considering different values of the fractional-order parameter $\alpha=0.5,1,1.5,2$, which depict weak, normal and strong conductivity at time $t=5 \mathrm{sec}$ for the thin circular plate subjected to the action of a moving heat source. Also, during plotting the value of $\varphi$ is kept for $\varphi=0 \& 2 \pi$.

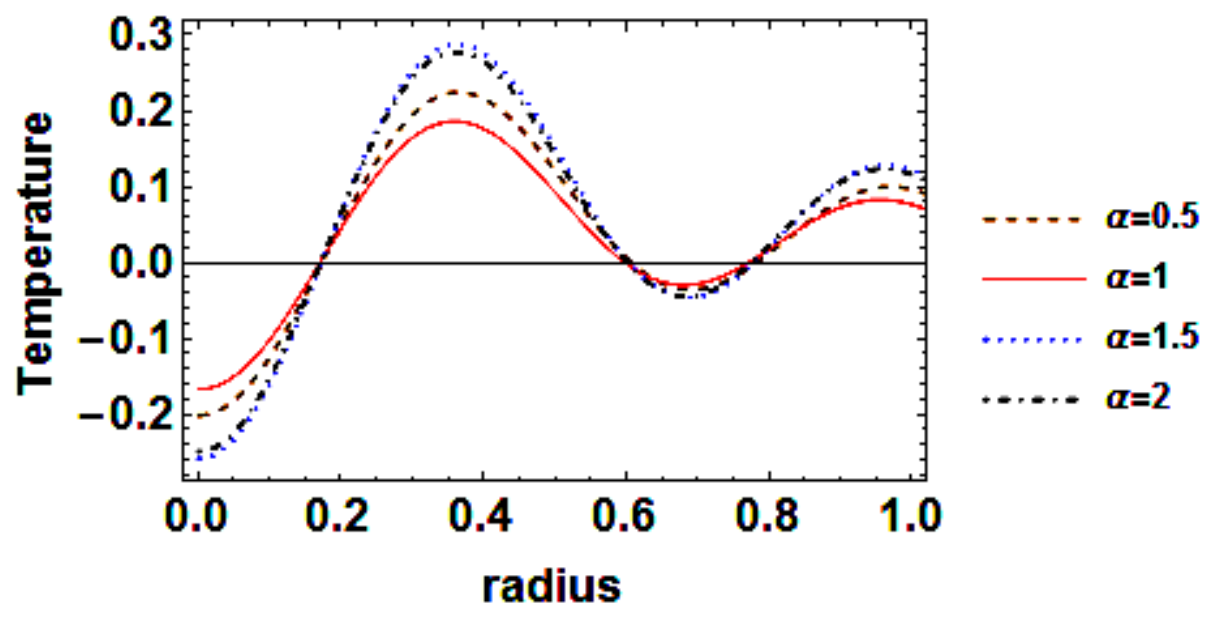

Fig.1. Temperature distribution versus radius.

Figure 1 shows the temperature distribution along the radial direction for the different values of the fractional-order parameter $\alpha=0.5,1,1.5,2$. It indicates that the temperature follow a nonuniform pattern with respect to the radius. The temperature increases gradually in the range $0 \leq r<0.4,0.7 \leq r<0.1$ and it decreases in the range $0.41 \leq r<0.69$. It is observed that the speed of propagation of thermal signals varies directly proportional to the fractional-order parameter $\alpha$. Small fluctuations of the temperature are identified near the centre of the plate, i.e. maximum heat is absorbed towards the center, it is due to the action of the moving heat source along a circular trajectory round the center of the plate with constant angular velocity.

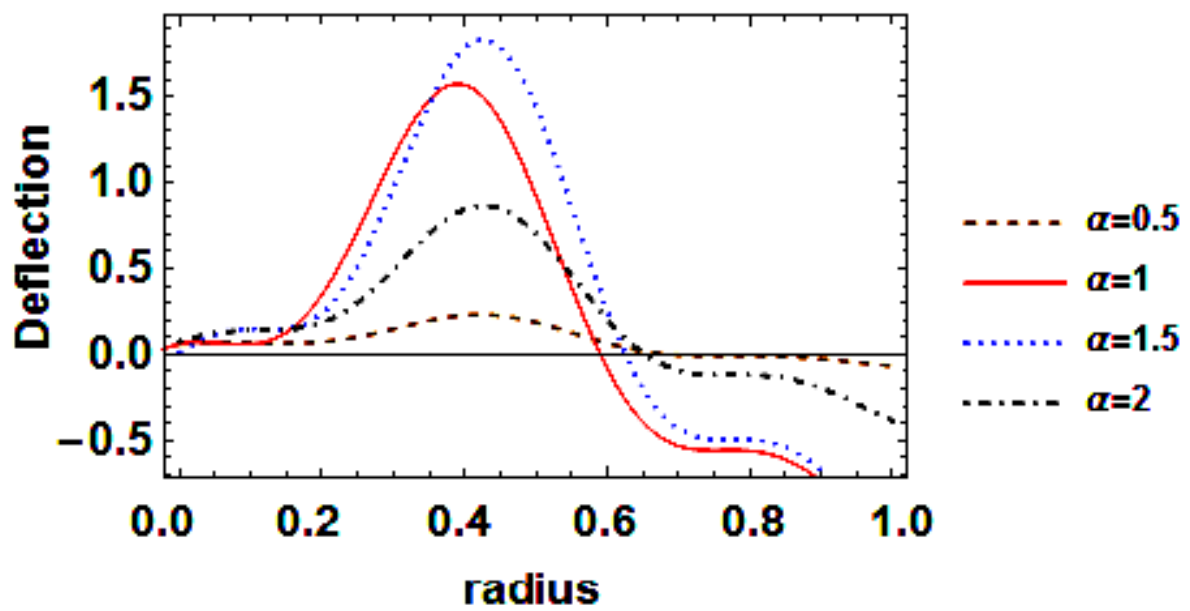

Fig.2. Thermal deflection versus radius.

Figure 2 shows of thermal deflection along the radial direction for the different values of the fractional-order parameter $\alpha=0.5,1,1.5$, 2. It can be observed that for all values of the fractional-order parameter the deflection gradually increases with an increase in the radius in the range $0 \leq r<0.5$ and flows in a nonuniform pattern and after mid-of-plate it starts decreasing gradually towards outer direction. Also, 
the deflection is zero at the initial radial ends $(r=0)$, which agrees with the prescribed boundary conditions. Further, it is observed that the fractional-order parameter $\alpha$ significantly affects thermal deflection. Hence it can be concluded that the fractional-order parameter is an important factor in the design of new materials.

\section{Conclusion}

In this article, we study the three dimensional temperature fields for a circular plate with the instantaneous point heat source $g(r, \varphi, z, t)$, which is situated inside the plate at the point $\left(r_{0}, \varphi(t), z_{0}\right)$. The thermal behaviour of the plate has been studied in the context of time fractional heat conduction by a quasi static approach using fractional calculus with physically convective heat exchange boundary conditions. An analytical solution is described by the integral transform technique and the corresponding thermal deflection was found using the thermal moments. It follows from the graphs that on changing values of the fractionalorder parameter $\alpha$, the speed of wave propagation is affected. Hence, it can be an important factor in the design of new materials applicable to real life situations and useful for researchers working in material sciences.

\section{Acknowledgment}

The authors sincerely thank the referees and Professor Pawel Jurczak, Editor-in-Chief, for their constructive comments which have improved the manuscript greatly.

\section{Nomenclature}

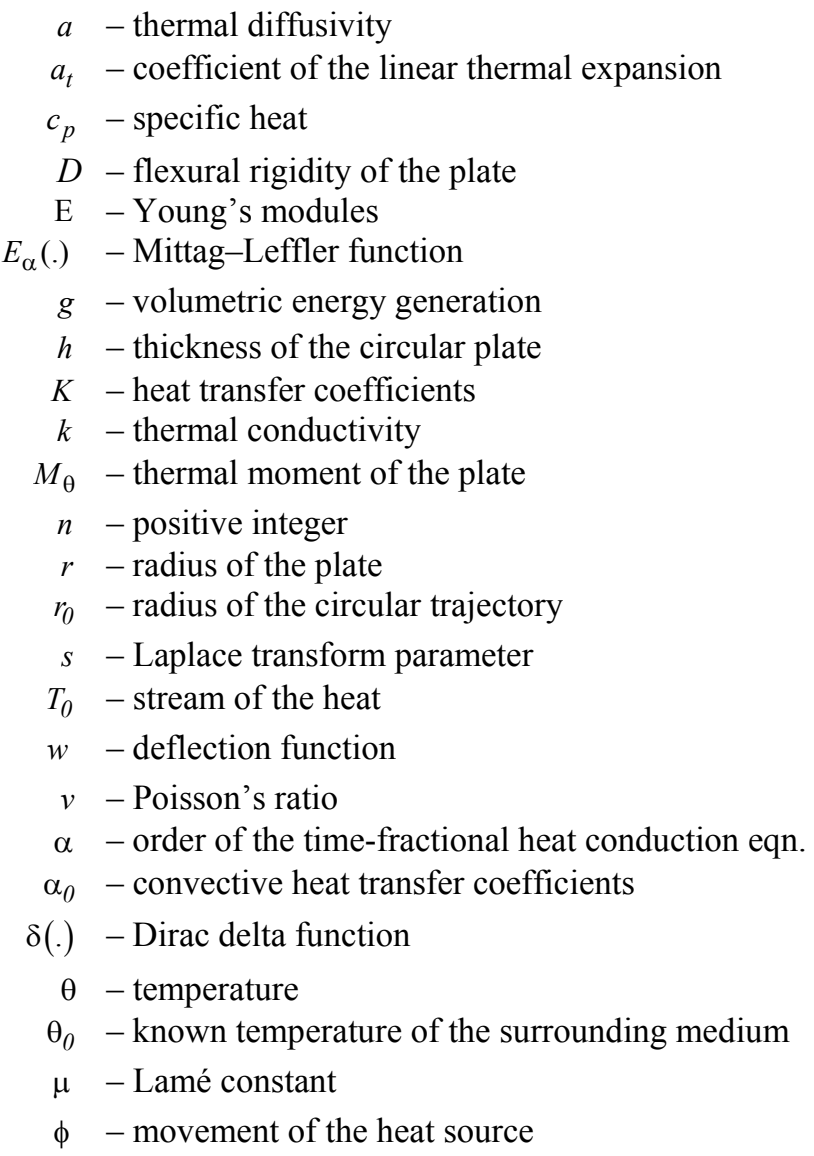


$\rho-$ density

$\omega-$ angular velocity of the moving heat source

\section{References}

[1] Povstenko Y.Z. (2005): Fractional heat conduction equation and associated thermal stresses. - J. Therm. Stress, vol.28, pp.83-102.

[2] Povstenko Y.Z. (2009): Thermoelasticity that uses fractional heat conduction equation. - Journal of Mathematical Stresses, vol.162, pp.296-305.

[3] Youssef H.M. (2010): Theory of fractional order generalized thermoelasticity. - J. Heat Transfer, vol.132, pp.1-7.

[4] Povstenko Y.Z. (2010): Fractional radial heat conduction in an infinite medium with a cylindrical cavity and associated thermal stresses. - Mech. Res. Commun, vol.37, pp.436-440.

[5] Jiang X. and Xu M. (2010): The time fractional heat conduction equation in the general orthogonal curvilinear coordinate and the cylindrical coordinate systems. - Physica A, vol.389, pp.3368-3374.

[6] Ezzat M.A. (2011): Magneto-thermoelasticity with thermoelectric properties and fractional derivative heat transfer. - Physica B, vol.406, pp.30-35.

[7] Povstenko Y.Z. (2011): Fractional Cattaneo-type equations and generalized thermoelasticity. - Journal of thermal Stresses, vol.34, No.2, pp.97-114.

[8] Ezzat M.A. (2011): Theory of fractional order in generalized thermoelectric MHD. - Applied Mathematical Modelling, vol.35, pp.4965-4978.

[9] Ezzat M.A. and Ezzat S. (2016): Fractional thermoelasticity applications for porous asphaltic materials. Petroleum Science, vol.13, No.3, pp.550-560.

[10] Ezzat M.A. and EI-Bary (2016): Modelling of fractional magneto-thermoelasticity for a perfect conducting materials.- Smart Structures and Systems, vol.18, No.4, pp.701-731.

[11] Xiong C. and Niu Y. (2017): Fractional order generalized thermoelastic diffusion theory.- Applied Mathematics and Mechanics, vol.38, No.8, pp.1091-1108.

[12] Khobragade N.L. and Kumar N. (2019): Thermal deflection and stresses of a circular disk due to partially distributed heat supply by application of fractional order theory. - Journal of Computer and Mathematical Sciences, vol.10, No.3, pp.429-437.

[13] Khobragade N.L. and Kumar N. (2019): Study of thermoelastic deformation of a solid circular cylinder by application of fractional order theory. - Journal of Computer and Mathematical Sciences, vol.10, No.3, pp.438-444.

[14] Khobragade N.L. and Lamba N.K. (2019): Magneto-thermodynamic stress analysis of an orthotropic solid cylinder by fractional order theory application. - Research and Reviews: Journal of Physics, vol.8, No.1, pp.37-45.

[15] Khobragade N.L. and Lamba N.K. (2019): Modeling of thermoelastic hollow cylinder by the application of fractional order theory. - Research and Reviews: Journal of Physics, vol.8, No.1, pp.46-57.

[16] Kidawa-Kukla J.(2008): Temperature distribution in a circular plate heated by a moving heat source. - Scientific Research of the Institute of Mathematics and Computer Science, vol.7, No.1, pp.71-76.

[17] Beck J.V., Cole K.D., Haji-Sheikh A. and Litkouhi B. (1992): Heat Conduction using Green's Functions. Hemisphere Publishing Corporation, Philadelphia.

[18] Kumar R., Lamba N.K. and Vinod V. (2013): Analysis of thermoelastic disc with radiation conditions on the curved surfaces. - Materials Physics and Mechanics, vol.16, pp.175-186.

[19] Lamba N.K. and Khobragade N.W. (2012): Integral transforms methods for inverse problem of heat conduction with known boundary of a thin rectangular object and its stresses. - Journal of Thermal Science vol.21, No.5, pp.459-465. 
[20] Lamba N.K. and Khobragade N.W. (2012): Uncoupled thermoelastic analysis for a thick cylinder with radiation. - Theoretical and Applied Mechanics Letters 2, 021005.

[21] Kamdi D. and Lamba N.K. (2016): Thermoelastic analysis of functionally graded hollow cylinder subjected to uniform temperature field. - Journal of Applied and Computational Mechanics, vol.2, No.2, pp.118-127.

[22] Noda N., Hetnarski R.B. and Tanigawa Y. (2003): Thermal Stresses. - Second Edition, Taylor and Francis, New York, pp.376-387.

[23] Sneddon I.N. (1972): The use of Integral Transforms. - New York: McGraw-Hill.

[24] Povstenko Y. (2015): Fractional Thermoelasticity. - New York: Springer.

[25] Hussain E.M. (2014): Fractional order thermoelastic problem for an infinitely long solid circular cylinder. Journal of Thermal Stresses, vol.38, pp.133-145.

[26] Raslan W. (2014): Application of fractional order theory of thermoelasticity to a 1d problem for a cylindrical cavity. - Arch. Mech., vol.66, pp.257-267.

[27] Hussain E.M. (2015): Fractional order thermoelastic problem for an infinitely long solid circular cylinder. Journal of Thermal Stresses, vol.38, pp.133-145.

[28] Caputo M. (1967): Linear model of dissipation whose Q is almost frequency independent-II. - Geophys. J. Royal Astron. Soc., vol.13, pp.529-935.

[29] Caputo M. (1974): Vibrations on an infinite viscoelastic layer with a dissipative memory. - J. Acoust. Soc. Am., vol.56, pp.897-904.

[30] Caputo M. and Mainardi F. (1971): A new dissipation model based on memory mechanism. - Pure Appl. Geophys., vol.91, pp.134-147.

[31] Caputo M. and Mainardi F. (1971): Linear model of dissipation in an elastic solid. - Rivista Del NuovoCimento, vol.1, pp.161-198.

Received: December 24, 2019

Revised: May 24, 2020 